\title{
Regeneration of Small Eddies by Data Assimilation in Turbulence
}

\author{
Kyo Yoshida, ${ }^{*}$ Junzo Yamaguchi $^{\dagger}$ and Yukio Kaneda \\ Department of Computational Science and Engineering, Graduate School of Engineering, Nagoya University, Chikusa-ku, \\ Nagoya 464-8603, Japan
}

(Received 27 August 2004; published 5 January 2005)

\begin{abstract}
The effect of data assimilation of large-scale eddies on small-scale eddies in turbulence is studied by direct numerical simulations (DNSs) of Navier-Stokes turbulence with Taylor microscale Reynolds numbers up to 179. The DNSs show that even if the data of small-scale eddies are lost at some initial instant, they can be regenerated from the data of large-scale eddies under the condition that Fourier modes with wave number less than a critical wave number $k^{*}$ are continuously assimilated, where $k^{*} \approx 0.2 \eta^{-1}$ with $\eta \equiv\left(\nu^{3} / \epsilon\right)^{1 / 4}, \epsilon$ the mean energy dissipation rate, and $\nu$ the viscosity.
\end{abstract}

It has been long and widely recognized that turbulence is sensitive to small differences in the flow conditions such as the boundary and initial conditions. For example, more than a half century ago, Batchelor [1] wrote in his book "some of these motions are such that the velocity at any given time and position in the fluid is not found to be the same when it is measured several times under seemingly identical conditions." The sensitivity is related to the limitation of the predictability of turbulence. In the context of weather predictions, Leith and Kraichnan [2] argued from a closure theoretic consideration that the predictability of the atmospheric turbulence is bounded within two weeks or so due to a small-scale error in the initial condition. Modern extensive studies of various nonlinear chaotic systems since Lorenz [3] have been reinforcing the view of sensitivity of turbulence to small errors or disturbances.

On the other hand, there is a possibility that turbulence may be insensitive to small errors under certain conditions. As a representative example, let us consider atmospheric turbulence. Although it is difficult to get observational data of atmospheric motion in full detail, it may be possible to get coarse grained (in space and time) information of the flow field, through, for example, satellite data. Charney et al. [4] proposed in meteorology a method called continuous data assimilation, in which observational data obtained at a coarse grained level are exploited in numerical models for the improvement in the estimate of the current atmospheric state.

In some recent studies [5-8], the idea of continuous data assimilation was applied to direct numerical simulations (DNSs) of Navier-Stokes turbulence. It was found that the error in flow field decreases; i.e., the flow field is insensitive to the initial error, when the coarse grained data at a certain level are used as the "boundary condition." This suggests that small eddies are subordinate to large eddies, in the sense that even if the data of small eddies are lost at some initial instant they can be "regenerated" by those of large eddies as time goes on. The problem of continuous data assimilation has been also studied from a mathematical point of view; it has a close link to the so-called theory of determining modes. (See, e.g., Refs. [9-11] and the references cited therein.)

In spite of these studies, little seems to be known about the quantitative aspects of the effect of data assimilation on turbulence, especially at high Reynolds number. In previous numerical studies, the Reynolds numbers have been too low to realize the so-called universal equilibrium range exhibiting the $k^{-5 / 3}$ energy spectrum. In mathematical studies, some conditions for effective data assimilation have been derived, but they seem too strong when compared to the results from the numerical experiments $[7,8]$.

In this Letter, we investigate the quantitative aspects of the effect of data assimilation on turbulence at high Reynolds number by DNSs. A particular stress is on the optimal estimate of the amount of data to be assimilated for the "regeneration" of the data of small-scale eddies, and also on the dependence of the amount on turbulence characteristics. For this purpose, we performed DNSs of threedimensional turbulence with Taylor microscale Reynolds number $R_{\lambda}$ in the range of 31-179. A quasi $k^{-5 / 3}$-power law of the energy spectrum which characterizes the inertial subrange is observed in the DNSs with the highest $R_{\lambda}$.

Let $\boldsymbol{u}^{(1)}(\boldsymbol{x}, t)$ and $\boldsymbol{u}^{(2)}(\boldsymbol{x}, t)$ be two three-dimensional incompressible turbulent flow fields obeying the same Navier-Stokes equations, but with different initial conditions and (possibly) different external forces, $\boldsymbol{f}^{(1)}(\boldsymbol{x}, t)$ and $\boldsymbol{f}^{(2)}(\boldsymbol{x}, t)$, respectively. For simplicity, we apply periodic boundary conditions in each of the Cartesian coordinates with period $2 \pi$. Let $\hat{\boldsymbol{u}}^{(i)}(\boldsymbol{k}, t)(i=1,2)$ be the Fourier transform of the velocity field $\boldsymbol{u}^{(i)}(\boldsymbol{x}, t)$ with respect to $\boldsymbol{x}$. The coarse grained data of $\boldsymbol{u}^{(1)}$ is assimilated to $\boldsymbol{u}^{(2)}$ by replacing $\hat{\boldsymbol{u}}^{(2)}(\boldsymbol{k}, t)$ by $\hat{\boldsymbol{u}}^{(1)}(\boldsymbol{k}, t)$ for low wave number modes satisfying $k \equiv|\boldsymbol{k}|<k_{a}$ at every time interval $T_{a}$ : hence $\hat{\boldsymbol{u}}^{(2)}\left(\boldsymbol{k}, t_{0}+n T_{a}\right)=\hat{\boldsymbol{u}}^{(1)}\left(\boldsymbol{k}, t_{0}+n T_{a}\right) \quad\left(k<k_{a}, n=\right.$ $1,2, \ldots)$. The limit $T_{a} \rightarrow 0$ corresponds to the continuous data assimilation. In this Letter, we consider only this limit unless otherwise stated. The velocity field $\boldsymbol{u}^{(1)}$ is a model of "true field," and $\boldsymbol{u}^{(2)}$ is a model of "simulated field with the use of coarse grained data of the true field." 
TABLE I. DNS parameters and turbulence characteristics of the initial velocity field $\boldsymbol{u}^{(1)}$ for each resolution. $N$ is the number of grid points along one of the Cartesian coordinates, $k_{\max }$ the maximum resolved wave number, $k_{i}$ the smallest $k$ such that $\Delta(k, 0) \neq 0, \nu$ the viscosity, $\Delta t$ the time increment, $\epsilon$ the mean energy dissipation rate, $L_{0}$ the integral length scale, $\lambda$ the Taylor microscale, $\eta$ the Kolmogorov length scale, and $R_{\lambda}$ the Taylor microscale Reynolds number.

\begin{tabular}{lrrrcrcccrr}
\hline \hline & $N$ & $k_{\max }$ & $k_{i}$ & $\nu\left(\times 10^{-3}\right)$ & $\Delta t\left(\times 10^{-3}\right)$ & $\epsilon$ & $L_{0}$ & $\lambda$ & $\eta\left(\times 10^{-3}\right)$ & $R_{\lambda}$ \\
\hline RUN64-1 & 64 & 30 & 28 & 10.0 & 10.0 & 0.171 & 0.941 & 0.541 & 49.2 & 31 \\
RUN128-1 & 128 & 60 & 58 & 2.70 & 10.0 & 0.138 & 0.796 & 0.312 & 19.4 & 67 \\
RUN256-1 & 256 & 120 & 112 & 1.10 & 4.00 & 0.132 & 0.759 & 0.204 & 10.0 & 107 \\
RUN512-1 & 512 & 241 & 236 & 0.410 & 1.00 & 0.127 & 0.728 & 0.127 & 4.82 & 179 \\
RUN128-2 & 128 & 60 & 58 & 5.50 & 10.0 & 0.148 & 0.882 & 0.431 & 32.6 & 45 \\
\hline \hline
\end{tabular}

Let $E^{(i)}(k, t)$ and $E^{(i)}(t)(i=1,2)$ be the energy spectra and the energies per unit mass of the velocity fields $\boldsymbol{u}^{(i)}(i=$ $1,2)$, respectively; similarly, let $\Delta(k, t)$ and $\Delta(t)$ be the energy spectrum and the energy of the difference (error) field, $\quad \delta \boldsymbol{u}=\boldsymbol{u}^{(2)}-\boldsymbol{u}^{(1)} ; \quad$ i.e, $\quad E^{(i)}(k, t)=$ $(1 / 2) \sum_{k^{\prime}=k}\left|\hat{\boldsymbol{u}}^{(i)}\left(\boldsymbol{k}^{\prime}, t\right)\right|^{2}, \quad E^{(i)}(t)=\sum_{k} E^{(i)}(k, t), \quad \Delta(k, t)=$ $(1 / 2) \sum_{k^{\prime}=k}\left|\delta \hat{\boldsymbol{u}}\left(\boldsymbol{k}^{\prime}, t\right)\right|^{2}$, and $\Delta(t)=\sum_{k} \Delta(k, t)$, where $\sum_{k^{\prime}=k}$ denote the summation over $k-1 / 2<\left|\boldsymbol{k}^{\prime}\right| \leq k+$ $1 / 2$. When $E^{(1)}(k, t)=E^{(2)}(k, t)$ is satisfied, the equality $\Delta(k, t)=0$ implies that $\hat{\boldsymbol{u}}^{(1)}=\hat{\boldsymbol{u}}^{(2)}$ in the wave number range $(k-1 / 2, k+1 / 2]$, whereas $\Delta(k, t)=2 E^{(1)}(k, t)$ implies that $\sum_{k^{\prime}=k} \hat{\boldsymbol{u}}^{(1)}\left(\boldsymbol{k}^{\prime}, t\right) \cdot \hat{\boldsymbol{u}}^{(2)}\left(-\boldsymbol{k}^{\prime}, t\right)=0$; i.e., $\boldsymbol{u}^{(1)}$ and $\boldsymbol{u}^{(2)}$ are uncorrelated in the wave number range. Thus, $\Delta(k, t)$ gives a quantitative measure of the difference between $\boldsymbol{u}^{(1)}$ and $\boldsymbol{u}^{(2)}$ in the wave number range. Similarly, $\Delta(t)$ gives a quantitative measure of the total error or difference.

We performed DNSs of $\boldsymbol{u}^{(1)}$ and $\boldsymbol{u}^{(2)}$ by using an aliasfree spectral method and a fourth-order Runge-Kutta method for the time marching. There are five groups of DNSs: RUN64-1, RUN128-1, RUN256-1, RUN512-1, and RUN128-2. Each group consists of several DNSs with the same number of grid points $N^{3}$, the same initial fields of $\boldsymbol{u}^{(1)}$ and $\boldsymbol{u}^{(2)}$, but with different $k_{a}$ 's.

The initial fields for $\boldsymbol{u}^{(1)}$ are statistically quasistationary states obtained by preliminary DNSs of a forced NavierStokes equation where the external force $f$ was applied in the low wave number range $2<k<3$ in the form of negative viscosity, such as $\hat{\boldsymbol{f}}(\boldsymbol{k}, t)=\gamma \hat{\boldsymbol{u}}(\boldsymbol{k}, t)$, and the value of $\gamma(>0)$ was determined at each time step so as to maintain the energy $E^{(1)}$ at an almost time-independent constant $(=0.5)$. The values of kinematic viscosity $\nu$ was so chosen that $k_{\max } \eta \approx 1$ (except for RUN128-2, in which $k_{\max } \eta \approx 2$ ), where $k_{\max }$ is the maximum wave number, $\eta \equiv\left(\nu^{3} / \epsilon\right)^{1 / 4}$ is the Kolmogorov length scale, and $\epsilon$ the mean energy dissipation rate per unit mass. The initial value of $\hat{\boldsymbol{u}}^{(2)}\left(\boldsymbol{k}, t_{0}\right)$ is same as that of $\hat{\boldsymbol{u}}^{(1)}\left(\boldsymbol{k}, t_{0}\right)$ for $k<k_{i}$, and $\hat{\boldsymbol{u}}^{(2)}\left(\boldsymbol{k}, t_{0}\right)$ for $k \geq k_{i}$ was generated randomly under the constraint that $E^{(2)}\left(k, t_{0}\right)=E^{(1)}\left(k, t_{0}\right)$ is almost satisfied, where $k_{i}$ is an arbitrary high wave number satisfying $k_{i}>$ $k_{a}$. Consequently, we have $\Delta\left(k, t_{0}\right)=0$ for $k<k_{i}$ and
$\Delta\left(k, t_{0}\right) \approx 2 E^{(1)}\left(k, t_{0}\right)$ for $k \geq k_{i}$; i.e., the initial error is localized at the high wave number range $k_{i} \leq k \leq k_{\max }$.

The limit $T_{a} \rightarrow 0$ was numerically approximated by $T_{a}=\Delta t$, where $\Delta t$ is the time increment of the time marching in the DNSs. In order to maintain quasistationary $E^{(i)}(k)(i=1,2)$, the external forces $\boldsymbol{f}^{(i)}$ were applied in a similar way as in the preliminary DNSs; i.e., $\boldsymbol{f}^{(i)}(\boldsymbol{k}, t)=$ $\gamma \boldsymbol{u}^{(i)}(\boldsymbol{k}, t)(2<k<3)$. We set $k_{a}$ to be larger than 3. Since, $\hat{\boldsymbol{f}}(\boldsymbol{k})=\mathbf{0}$ for $k \geq k_{a}$ and $\delta \hat{\boldsymbol{u}} \neq \mathbf{0}$ only for $k \geq k_{a}, \boldsymbol{f}$ has no direct influence on $\delta \boldsymbol{u}$; i.e., all the influence of $\boldsymbol{f}$ on $\delta \boldsymbol{u}$ is through nonlinear terms of $\boldsymbol{u}$.

In Table I, some turbulence characteristics of the initial field $\boldsymbol{u}^{(1)}\left(\boldsymbol{k}, t_{0}\right)$, which are almost the same as those of $\boldsymbol{u}^{(2)}\left(\boldsymbol{k}, t_{0}\right)$, are listed together with the DNSs parameters. Here, the integral length scale $L_{0}=\left(\pi / 2 u^{\prime 2}\right) \times$ $\int d k k^{-1} E(k)$, the Taylor microscale $\lambda=\left(15 \nu u^{\prime 2} / \epsilon\right)^{1 / 2}$, and the Taylor microscale Reynolds number $R_{\lambda}=$ $\left(u^{\prime} \lambda / \nu\right)$, where $u^{\prime}=(2 E / 3)^{1 / 2}$.

Figure 1 shows the initial energy spectra $E^{(1)}(k)$ $\left[\approx E^{(2)}(k)\right]$ at $t=t_{0}$. Note that quasi $k^{-5 / 3}$ spectrum which characterize inertial subrange statistics is observed in the

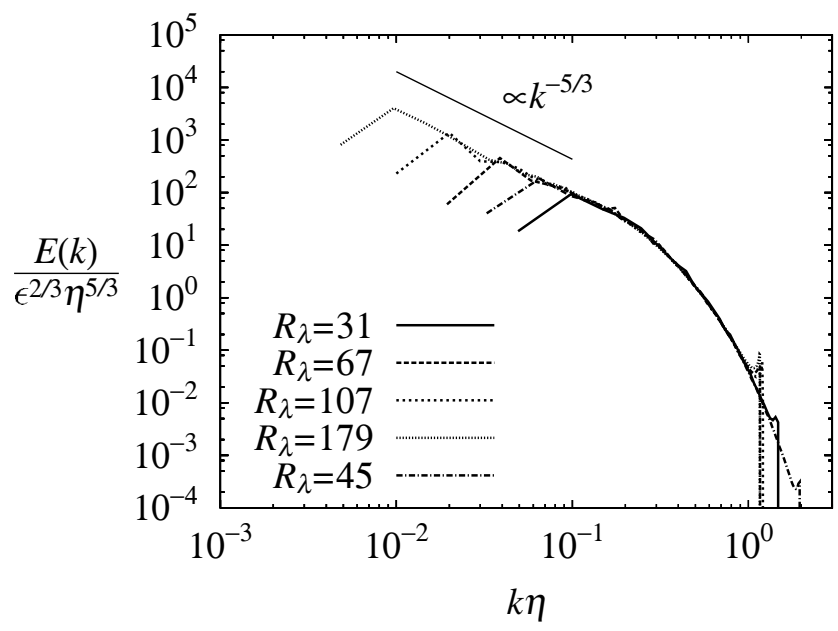

FIG. 1. Energy spectra $E^{(1)}(k)$ of the initial velocity fields $\boldsymbol{u}^{(1)}$ 's for various $R_{\lambda}$ 's. Both axes are nondimensionalized by the energy dissipation rate $\epsilon$ and the Kolmogorov length scale $\eta$. 


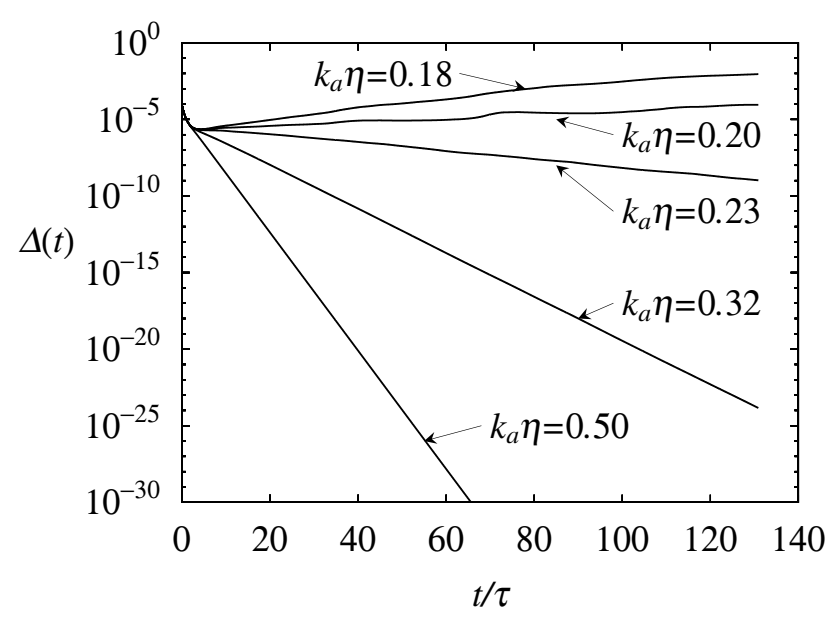

FIG. 2. Evolution of $\Delta(t)$, the energy of the difference field $\boldsymbol{u}^{(2)}-\boldsymbol{u}^{(1)}$, for various $k_{a}$ 's in the simulations with $R_{\lambda}=107$.

simulation with the highest Reynolds number $R_{\lambda}=179$ $(N=512)$.

Figure 2 shows $\Delta(t)$ versus nondimensional time $t / \tau$, for $R_{\lambda}=107$ and some $k_{a}$ 's, where the initial time is set to $t_{0}=0$, and $\tau$ is the Kolmogorov time scale defined by $\tau \equiv$ $(\nu / \epsilon)^{1 / 2}$. If $k_{a}$ is low, $\Delta(t)$ increases with time after some initial transient period. This is consistent with the idea of the sensitivity of turbulence to the initial condition, known in literature (see, e.g., Ref. [2]). However, $\Delta(t)$ decreases with time if $k_{a}$ is higher than a certain wave number.

The figure shows that $\Delta(t)$ increases or decreases approximately in an exponential form, i.e.,

$$
\Delta(t) \approx A \exp (-\alpha t)
$$

after some initial transient period, where the decay constant $\alpha$ depends on $k_{a}$. Let $\bar{\alpha}$ and $\Delta \alpha$ be the average and the standard deviation, respectively, of the local decay constant $\alpha(t) \equiv-\partial / \partial t[\log \Delta(t)]$ over a time interval $10 \tau<t \leq$ $T_{\max }$, where $T_{\max }$ is either of the time that the DNS was terminated, the time at which $\Delta(t)$ become smaller than $10^{-30}$ (when $\alpha>0$ ), or an arbitrary time well before $\Delta(t)$ stops growing exponentially and saturates (when $\alpha<0$ ). The initial period $0 \leq t \leq 10 \tau$ is not included here in the time average.

We confirmed that the difference between $\bar{\alpha}$ 's with $T_{a}=$ $\Delta t$ and $T_{a}=2 \Delta t$ is typically $\sim 1 \%$. Thus, $T_{a}=\Delta t$ may be a good approximation for the limit $T_{a} \rightarrow 0$ in estimating $\bar{\alpha}$. The averaged decay constant $\bar{\alpha}$ is almost independent of $k_{i}$ as far as there exists a long period of exponential decay (or growth). For given $k_{a}$, a typical difference of $\bar{\alpha}$ among DNSs with different $k_{i}$ 's is smaller than $\Delta \alpha$.

The averaged decay constant $\bar{\alpha}\left(k_{a}\right)$ as a function of $k_{a}$ varies with $R_{\lambda}$. However, it is found that the nondimensional function $\tilde{\alpha}$ defined by

$$
\tilde{\alpha}\left(k_{a} \eta\right)=\bar{\alpha}\left(k_{a}\right) \tau
$$

is quite insensitive to $R_{\lambda}$, as shown in Fig. 3. Let $k^{*}$ be the critical wave number such that $\Delta(t)$ decreases with time if and only if $k_{a}>k^{*}$ [i.e., $\bar{\alpha}\left(k^{*}\right)=0$ in Fig. 3]. One can estimate from Fig. 3 that

$$
k^{*} \approx 0.2 \eta^{-1} .
$$

Note that data from DNSs with $k_{\max } \eta \approx 1$ (RUN64-1, RUN128-1, RUNS256-1, and RUN512-1) and those from DNSs with $k_{\max } \eta \approx 2$ (RUN128-2) collapse well. This fact suggests that $k_{\max } \eta \approx 1$ is a sufficient resolution for the estimate of $\bar{\alpha}$. Our preliminary study suggests that $\bar{\alpha}$ by DNS with $k_{\max } \eta<1$ is fairly smaller than those in Fig. 3 (figure omitted), so that $k_{\max } \eta \geq 1$ is necessary for the proper estimate of $\bar{\alpha}$.

We may interpret the averaged decay constant $\bar{\alpha}$ as a function of the energy ratio $E_{a} / E$, or as a function of the enstrophy ratio $\Omega_{a} / \Omega$, where $E_{a}=\sum_{k<k_{a}} E(k), \Omega_{a}=$ $\sum_{k<k_{a}} k^{2} E(k)$, and $\Omega=\sum_{k} k^{2} E(k)$. Figure 4(a) shows the nondimensional decay constant $T_{e} \bar{\alpha}$ as a function of $E_{a} / E$, for various $R_{\lambda}$ 's, where $T_{e}$ is the eddy turnover time defined by $T_{e} \equiv L_{0} / u^{\prime}$. One can see from the figure the critical ratio $\left(E_{a} / E\right)^{*}$ for which $\bar{\alpha}=0$ strongly depends on $R_{\lambda}$. On the other hand, Fig. 4(b) shows that the curves of the nondimensional decay constant $\tau \bar{\alpha}(=\tilde{\alpha})$ collapse well for various $R_{\lambda}$ 's, when they are plotted as functions of $\Omega / \Omega_{a}$. The figure suggests that $0.30 \lesssim\left(\Omega_{a} / \Omega\right)^{*} \lesssim 0.35$, where $\left(\Omega_{a} / \Omega\right)^{*}$ is the critical ratio for which $\bar{\alpha}=0$. We may conclude from Figs. 3 and 4 that $\bar{\alpha}$ is well characterized by the quantities for the energy dissipation range, such as $\eta, \tau$, and $\Omega$, rather than those for the energy containing range, such as $L_{0}, T_{e}$, and $E$.

Let $\operatorname{Re}_{\ell} \equiv u_{\ell} \ell / \nu$ be the Reynolds number associated with eddies of size $\ell$, where $u_{\ell}$ is the characteristic velocity of eddies of size $\ell$. The use of the Kolmogorov scaling

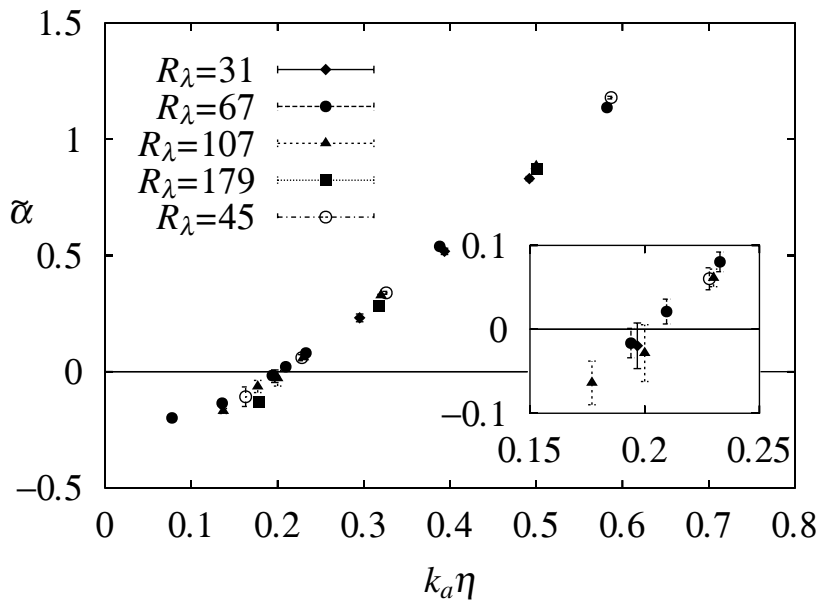

FIG. 3. Nondimensional decay constant $\tilde{\alpha}$ versus $k_{a} \eta$ for various $R_{\lambda}$ 's. The error bars show the nondimensional standard deviations $\tau \Delta \alpha$. The inset shows a close-up view of a range where $\tilde{\alpha}$ changes the sign. 

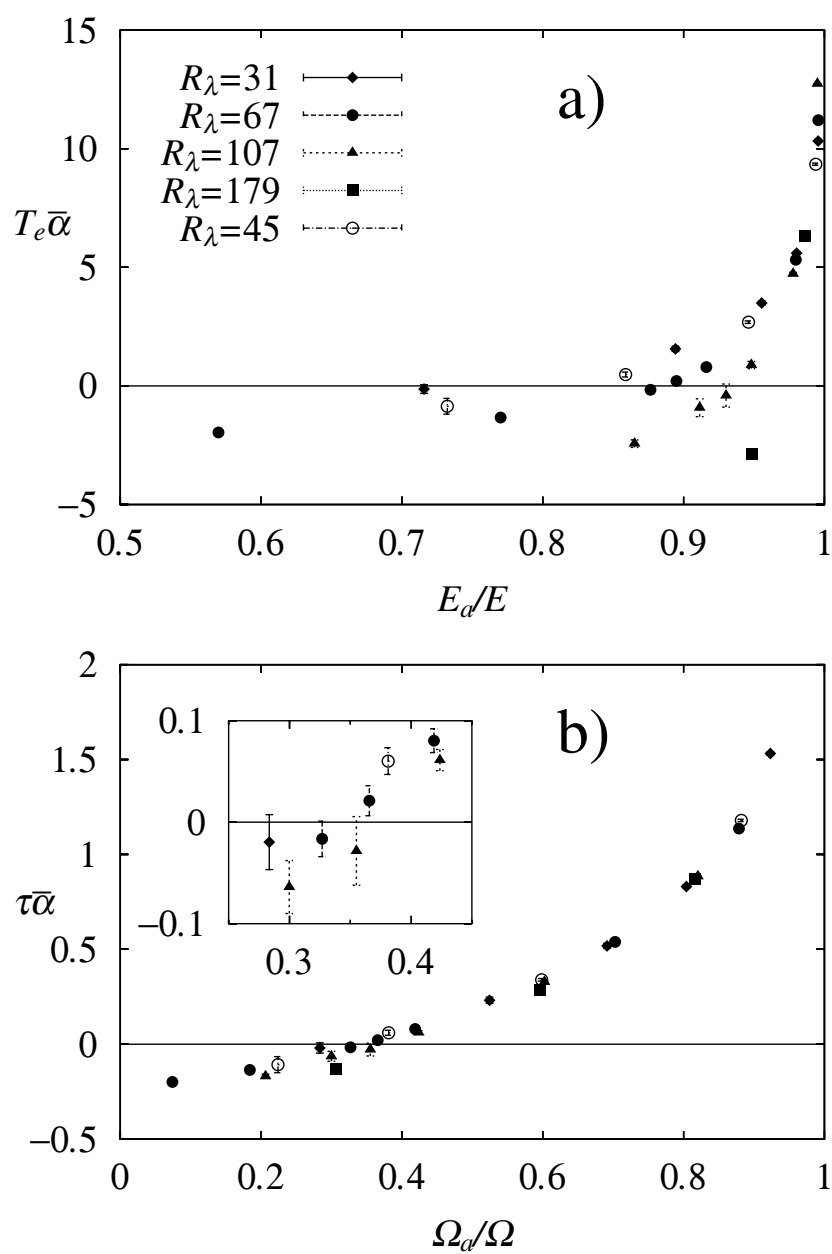

FIG. 4. (a) Nondimensional decay constant $T_{e} \bar{\alpha}$ versus energy ratio $E_{a} / E$ for various $R_{\lambda}$ 's. The error bars show the nondimensional standard deviations $T_{e} \Delta \alpha$. (b) Nondimensional decay constant $\tau \bar{\alpha}(=\tilde{\alpha})$ versus enstrophy ratio $\Omega_{a} / \Omega$. The symbols denote the values of $R_{\lambda}$ as shown in (a). The inset shows a closeup view of a range where $\tau \bar{\alpha}$ changes the sign.

$u_{\ell} \sim(\epsilon \ell)^{1 / 3}$ gives $\operatorname{Re}_{\ell} \sim(\ell / \eta)^{4 / 3}$, so that $\operatorname{Re}_{1 / k^{*}} \sim$ $\left(k^{*} \eta\right)^{-4 / 3} \sim 9$ for the eddies of size of $\ell^{*}=1 / k^{*}$. It is tempting to regard this "critical" Reynolds number $\operatorname{Re}_{1 / k^{*}}$ as an analogue of the well known critical Reynolds number $\mathrm{Re}_{c}$ for flows under given boundary conditions (for example, $\operatorname{Re}_{c}=5722$ for the plane Poiseuille flow), although the boundary conditions are not stationary, but determined by time-dependent turbulent large-scale flows, in the present case.

In conclusion, the DNSs show that $\tilde{\alpha}$ is insensitive to $R_{\lambda}$ within the range of the present simulations. The DNSs also suggest that if and only if $k_{a}>k^{*}$, where $k^{*} \approx 0.2 \eta^{-1}$, the data of high wave number modes with $k \geq k_{a}$ can be regenerated from those of low wave number modes with $k<k_{a}$. As far as this condition is satisfied, the flow field is insensitive to initial errors or uncertainties, in contrast to the widely spread view of the sensitivity of turbulence or nonlinear chaotic systems to small disturbances or errors. Note that the number of the Fourier modes satisfying $k<$ $0.2 \eta^{-1}$ is much smaller than that of the total modes. For example, it is only less than $1 \%$ of the total modes in DNSs with $k_{\max } \eta=1$. Thus only data of a very small portion of the total modes are necessary for the regeneration of the data of high wave number modes used in the DNSs.

The authors are grateful to L. Skrbek and E. S. Titi for useful discussions. The DNSs were performed on the Fujitsu VPP5000/56 system at the Information Technology Center of Nagoya University. This work has been partially supported by a Grant-in-Aid for the 21 st Century COE "Frontiers of Computational Science," and a Grant-in-Aid for Exploratory Research 14654073 from the Ministry of Education, Culture, Sports, Science, and Technology of Japan, and also by Grants-in-Aid for Scientific Research (B) 14340033 and (C) 15607011 from the Japan Society for the Promotion of Science.

*Present address: Department of Physics, Graduate School of Pure and Applied Sciences, University of Tsukuba, 1-11 Tennoudai, Tsukuba, Ibaraki 305-8671, Japan.

Electronic address: yoshida@sakura.cc.tsukuba.ac.jp

${ }^{\dagger}$ Present address: Denso Corporation, 1-1 Showa-cho, Kariya 448-8661, Japan.

[1] G. K. Batchelor, The Theory of Homogeneous Turbulence (Cambridge University Press, Cambridge, UK, 1953), Chap. 1.

[2] C. E. Leith and R. H. Kraichnan, J. Atmos. Sci. 29, 1041 (1972).

[3] E. N. Lorenz, J. Atmos. Sci. 20, 130 (1963).

[4] J. Charney, H. Halem, and R. Jastrow, J. Atmos. Sci. 26, 1160 (1969).

[5] G. Browning, W. Henshaw, and H. Kreiss, UCLA CAM Technical Report No. 98-23, 1998.

[6] K. Hayashi, T. Ishihara, and Y. Kaneda, in Statistical Theories and Computational Approaches to Turbulence, Modern Perspectives and Applications to Global-Scale Flows, edited by Y. Kaneda and T. Gotoh (Springer, New York, 2002), p. 239.

[7] W. Henshaw, H.-O. Kreiss, and J. Yström, Multiscale Modeling Sim. 1, 119 (2003).

[8] E. Olson and E. S. Titi, J. Stat. Phys. 113, 799 (2003).

[9] C. Foias and G. Prodi, Rend. Sem. Mat. Univ. Padova 39, 1 (1967).

[10] C. Foias, O.P. Manley, R. Temam, and Y.M. Trève, Physica (Amsterdam) 9D, 157 (1983).

[11] D. A. Jones and E. S. Titi, Ind. Univ. Math. J. 42, 875 (1993). 